\title{
Hamiltonian Mechanical System with Split Quaternions
}

\author{
Cansel YORMAZ ${ }^{1}$, Serife Naz ELMAS, Simge SIMSEK
}

Department of Mathematics, Pamukkale University, Turkey

Copyright (c) 2018 by authors, all rights reserved. Authors agree that this article remains permanently open access under the terms of the Creative Commons Attribution License 4.0 International License

\begin{abstract}
In this article, firstly we study about geometrical applications of split quaternions. Then, we obtain Hamitonian mechanical systems with Split quaternions. Quaternionic and Coquaternionic (split analoque of quaternions) extensions of Hamiltonian mechanics are introduced and are shown as offer a unifying framework for quantum mechanics. This study leads to the possibility of employing algebraic techniques of quaternions and coquaternions to absorbing in quantum mechanics. The founded equations are compared with the Hamiltonian energy equations generally are known and the Hamilton energy equations are obtained in Minkowski space.
\end{abstract}

Keywords Hamiltonian Mechanical System, Minkowski Space, Jet bundles, Hamiltonian Energy Equations, Quaternion, Split Quaternion

\section{Introduction}

Many of problems in classical mechanics may be solved based on Hamiltonian energy equations using Euclidian space, but none of them are calculated with quaternions. Quaternionic structure of a space is similar to Minkowski space. For mechanical systems, solving problems with jet bundles is convenient. Therefore, this study contains quaternionic jet bundles. In this study, we prefer to solve Hamiltonian energy equations with quaternions on a jet bundle structure. On the other hand, Sir William Rowan Hamilton introduced the set of quaternions in 1843, which can be presented as

$$
\mathbf{H}=\left\{q=q_{0}+q_{1} i+q_{2} j+q_{3} k, q_{0}, q_{1}, q_{2}, q_{3} \in R\right\}
$$

where

$$
i^{2}=j^{2}=k^{2}=-1 \quad \text { and } \quad i j k=-1
$$

After the work of Hamilton in 1849, James Cockle introduced the set of split quaternions, which can be represented as

$$
\hat{\mathbf{H}}=\left\{q=q_{0} 1+q_{1} i+q_{2} j+q_{3} k, q_{0}, q_{1}, q_{2}, q_{3} \in R\right\}
$$

where

$$
i^{2}=-1 \quad, \quad j^{2}=k^{2}=1 \quad \text { and } \quad i j k=1
$$

Since quaternions are non- commutative, they are not the same with complex numbers and real numbers. The set $\hat{\mathbf{H}}$ is non- commutative, too. But, $\hat{\mathbf{H}}$ contains zero divisors, nilpotent elements and non trivial idempotents.

Until the middle of the 20. century, the practical use of quaternions has been minimal in comparison with other method. But, currently, this situation has changed due to progress in robotics, animation and computer graphics technology. Also, quaternions are an efficient way understanding many study of physics and kinematics. Today, quaternions are used especially in the problems involving the estimation of rigid body transformations. 
Previous studies, Aycan [1,2] improved Hamiltonian energy equations on jet bundles and complex jet bundles. Daglı [8] improved Hamiltonian energy systems on Minkowski space. Sardanashvily $[9,10]$ has studied time dependent Hamiltonian systems and Hamiltonian systems with fibred manifold. The article [3] showed the basic geometrical structures for mechanical systems. In the studies[3,4] have been obtained algebraic structures of quaternions. In this study, we calculate Hamiltonian energy equations with quaternions. Hamiltonian systems are characterized by the cotangent bundle which are phase space of velocities and momentum of a configuration manifold.

Let us we take $M$ is a m-dimensional manifold and $\phi$ is a 2-form on the tangent bundle $T M$. If $\phi$ is a closed 2-form with maximal rank, the binary $(M, \phi)$ can be defined as a symplectic manifold. Also, the energy function $H: M \rightarrow R$ which response to the Lagrangian function, is named as Hamiltonian energy function and it is formulated as below;

$$
H=H\left(x_{i}, \dot{x}_{i}, t\right)=\left\langle x_{i}, \ddot{x}_{i}\right\rangle-L\left(x_{i}, \ddot{x}_{i}, t\right)
$$

Let the coordinate system on $M$ is $\left(x_{i}, \dot{x}_{i}\right)$, then the vector field

$$
X_{H}=\frac{\partial H}{\partial \dot{x_{i}}} \frac{\partial}{\partial x_{i}}-\frac{\partial H}{\partial x_{i}} \frac{\partial}{\partial \dot{x}_{i}}
$$

can defined as Hamiltonian vector field. The equation

$$
i_{X_{H}} \phi=d H
$$

is named of the dynamical equation for Hamiltonian system. On the contangent bundle $T^{*} M$, the 1 - form

$$
\lambda_{M}=\dot{x}_{i} d x_{i}
$$

is named as Liouville form, then the 2 -form,

$$
\phi_{M}=-\lambda_{M}=d x_{i} \wedge d \dot{x}_{i}
$$

is named as canonical symplectic form. With solving equations 1.2 , we can obtain equations

$$
\frac{\partial x_{i}}{\partial t}=\frac{\partial H}{\partial \dot{x}_{i}}, \quad \frac{\partial \dot{x_{i}}}{\partial t}=-\frac{\partial H}{\partial x_{i}}
$$

These are Hamiltonian equations of motion. Although, they are strictly equivalent to Lagrangian and Newton equations, the equations of Hamilton have proven to be more suitable for adaptation to quantum mechanics. The Hamiltonian formulations of mechanics are also notable for the fact that they express the laws of mechanics without reference to any particular coordinate system for the configuration space. Of course, in their original forms, they assumed an absolute time coordinate and perfectly rigid bodies, but with suitable restrictions they can be adapted to relativistic mechanics as well. In quantum mechanics, a pair of conjugate variables $\left(x_{i}, \dot{x}_{i}\right)$, such as position and momentum, generally do not commute, which means that the operation consisting of a measurement of $x_{i}$ followed by a measurement of $x_{i}$ is different than the operation of performing these measurements in the reverse order. This is because the eigenstates corresponding to the respective measurement operators are incompatible. As a result, the system cannot simultaneously have both a definite value of $\dot{x}_{i}$ and a definite value of $x_{i}$.

Inclusion of time dimension for solving mechanical systems is an important parameter that easy to solve Hamiltonian equations. In order to improve the Hamiltonian systems on this manifolds, we propose to take time derivatives for the take on jet bundles. If the bundle includes time dimension it leads to a Hamiltonian mechanical systems.

Quaternions could be viewed as the exterior tensor product of a scalar and a 3_dimensional vector. Under rotation for an event in spacetime represented by a quaternion, time is unchanged, but the 3_dimensional vector for space would be rotated. The treatment of scalars are the same as above, but the notion of vectors is far more restrictive the notion of scalars. Quaternions can only handle 3_dimensional vector to those familiar to playing with higher dimensions, this may appear too restrictive to be of interest. Yet physics on both the quantum and cosmological scales is confined to three spatial dimensions. The metric for quaternions is embedded in Hamiltonian rule for the field.

$$
i^{2}=j^{2}=k^{2}=-1 \quad \text { and } \quad i j k=-1
$$

This looks like a way to generate scalars from vectors, but it is more than that. It also says implicitly that $i j=k, j k=i$ and $i, j, k$ must be invertible. This is an important observation, because it means that inner and exterior tensor products can occur in the same operation.

For calculating with only information contained in events requires that a scalar and a 3_dimensional vector form for a field. According to a theorem by Frobenius on finite dimensional fields, the only fields that fit are isomorphic to the 
quaternions (isomorphic is a sophisticated notion of equality, whose subtleties are appreciated only by people with a deep understanding of mathematics). To do calculus with a mass or an electromagnetic potential has an identical requirement and an identical solution. This is the logical foundation for doing physics with quaternions. Can physics be done without quaternions; yes it can. Events can be defined over the field of real numbers, and then the Minkowski metric can be used to get every result ever confirmed by experiment.

Consider a set of quaternions that represent events in spacetime. If the magnitude of the 3_dimensional space vector is less than the time scalar, events are separated by a timelike interval. It requires a speed less than the speed of light to connect the events. This is true no matter what coordinate system is chosen. Every quaternion can be written in terms of its basis components,

$$
q=\left(q_{0}, q_{1}, q_{2}, q_{3}\right)=q_{0} 1+q_{1} i+q_{2} j+q_{3} k
$$

with addition defined pairwise and multiplication defined by the following rules:

$i, j$ and $k$ are all square roots of $-1 . i j=k=-j i ;-j k=i=k j ; k i=j=-i k$. Check that these relations $(i j=k=-j i$, etc.) all follow from Hamiltonian definition:

$$
i^{2}=j^{2}=k^{2}=i j k=-1
$$

Quaternions may also be written as an ordered pair of a scalar and a vector,

$$
q=\left(q_{0}, \vec{q}_{V}\left(q_{1}, q_{2}, q_{3}\right)\right)
$$

A quaternion with only a scalar part is called a real quaternion and a quaternion with only a vector part is called a pure quaternion.

According to the structure of split quaternion, this time parameter consist of scalar and vector parts as follow;

$$
t=t_{0}+i t_{1}+j t_{2}+k t_{3}=t_{s}+t_{v}
$$

On the other hand, this system is considered as split quaternion. Then, the Hamiltonian energy systems are obtained with split quaternion.

Definition 1. (Split Quaternion)

Split quaternion algebra is an associative, non-commutative, non-division ring with four basic elements $\{1, i, j, k\}$ satisfying the following equalities;

$$
\begin{aligned}
i^{2}=-1, \quad j^{2} & =k^{2}=1 \quad \text { and } \quad i j k=1 \\
i j & =-j i=k \\
k j & =-j k=-i, \\
k i & =-i k=j
\end{aligned}
$$

The set of split quaternions can be represented as;

$$
\hat{\mathbf{H}}=\left\{q=q_{0} 1+q_{1} i+q_{2} j+q_{3} k, q_{0}, q_{1}, q_{2}, q_{3} \in R\right\}
$$

Also, we write any split quaternion in the form

$$
q=\left(q_{0}, q_{1}, q_{2}, q_{3}\right)=S_{q}+\vec{V}_{q}
$$

where $S_{q}=q_{0}$ denotes the scalar part of $q$ and $\vec{V}_{q}=q_{1} i+q_{2} j+q_{3} k$ denotes vector part of $q$. If $S_{q}=q_{0}$ then $q$ is called pure split quaternion and the set of pure split quaternion can be identified with Minkowski 3_ space. The split quaternion product of two split quaternions $q=\left(a_{0}, a_{1}, a_{2}, a_{3}\right)$ and $p=\left(b_{0}, b_{1}, b_{2}, b_{3}\right)$ is defined as

$$
q * p=S_{q} S_{p}+\left\langle V_{q}, V_{p}\right\rangle+S_{q} V_{p}+S_{p} V_{q}+V_{q} \times_{L} V_{p}
$$

where $\langle$,$\rangle and \times_{L}$ are Lorentzian inner product and vector (exterior) product respectively. Also, the split quaternion product may be written as

$$
q * p=\left[\begin{array}{cccc}
a_{0} & -a_{1} & a_{2} & a_{3} \\
a_{1} & a_{0} & a_{3} & -a_{2} \\
a_{2} & a_{3} & a_{0} & -a_{1} \\
a_{3} & -a_{2} & a_{1} & a_{0}
\end{array}\right]\left[\begin{array}{l}
b_{0} \\
b_{1} \\
b_{2} \\
b_{3}
\end{array}\right]
$$


Let $q=\left(q_{0}, q_{1}, q_{2}, q_{3}\right)=S_{q}+\vec{V}_{q}$ be a split quaternion. The conjugate of a split quaternion, denoted $K_{q}$, is defined as $K_{q}=S_{q}-\vec{V}_{q}$. Since the vector parts of $q$ and $K_{q}$ differ only in sign, we have $I_{q} \stackrel{\text { def }}{=} q * K_{q}=K_{q} * q$. Now, we can define timelike, spacelike and lightlike quaternions, since the set of split quaternions, since the set of split quaternions $\hat{\mathbf{H}}$ is identified with semi- Euclidean space $E_{2}^{4}$.

Definition 2. We say that ;

i) If $I_{q}<0$ then a split quaternion $q$ is spacelike quaternion

ii) If $I_{q}>0$ then a split quaternion $q$ is timelike quaternion

iii) If $I_{q}=0$ then a split quaternion $q$ is lightlike quaternion

where $I_{q} \stackrel{\text { def }}{=} q * K_{q}=K_{q} * q$. Obviously, $-I_{q}=-\left(a_{0}\right)^{2}-\left(a_{1}\right)^{2}+\left(a_{2}\right)^{2}+\left(a_{3}\right)^{2}$ is identified with $\langle q, q\rangle_{E_{2}^{4}}$ for split quaternion $q=\left(q_{0}, q_{1}, q_{2}, q_{3}\right)$. [7]

Definition 3. The norm of $q=\left(q_{0}, q_{1}, q_{2}, q_{3}\right)$ is defined as

$$
N_{q}=\sqrt{\left|\left(q_{0}\right)^{2}+\left(q_{1}\right)^{2}-\left(q_{2}\right)^{2}-\left(q_{3}\right)^{2}\right|}
$$

If $N_{q}=1$ then $q$ is called unit split quaternion and $q_{0}=\frac{q}{N_{q}}$ is a unit split quaternion for $N_{q} \neq 0$. Also, spacelike and timelike quaternions have multiplicative inverses and they hold the property $q * q^{-1}=q^{-1} * q=1$. And they are constructed by $q^{-1}=\frac{K_{q}}{I_{q}}$. Lightlike quaternions have no inverses.

Theorem 1. Split quaternions satisfy the following properties;

i) $\quad q *(r * s)=(q * r) * s$

ii) $q *(r+s)=q * r+q * s$

iii) $K_{q * r}=K_{r} * K_{q}$

iv) $I_{q * r}=I_{q} I_{r}$

v) $N_{q * r}=N_{q} N_{r}$

vi) $V_{q}$ is parallel to $V_{r}$ if and only if $\forall q, r, s \in \hat{\mathbf{H}}$, for $q * r=r * q .[7]$

The set of timelike quaternions denoted by

$$
T \hat{\mathbf{H}}=\left\{q=\left(a_{0}, a_{1}, a_{2}, a_{3}\right): a_{0}, a_{1}, a_{2}, a_{3} \in R, I_{q}>0\right\}
$$

forms a group under the split quaternion product. Also, the set of unit timelike quaternions represented by $T \hat{\mathbf{H}}_{1}$ and identified with semi- Euclidean sphere $S_{2}^{3}=\left\{u \in E_{2}^{4}:\langle u, u\rangle_{E_{2}^{4}}=1\right\}$ is a subgroup of $T \hat{\mathbf{H}}$ [7]

\section{SPLIT QUATERNIONIC BUNDLES ON MINKOWSKI 4- SPACE}

Let $(E, \pi, M)$ is a bundle where, $E$ and $M$ are manifolds and $\pi: E \rightarrow M$ is a surjective submersion. $E$ is called the total space, $\pi$ is the projection and $M$ is the base space. This bundle denoted by $\pi$ or $E$. The first jet manifold of $\pi$ is the set $\left\{J_{p}^{1} \phi: p \in M, \phi \in \Gamma_{p}(\pi)\right\}$ and denoted by $J^{1} E$. Here, $\phi=M \rightarrow E$. $\phi$ is a map and called as section of $\pi$. If it is satisfies the condition $\pi \circ \phi=i d_{M}$, then the set of all sections of $\pi$ will be denoted $\Gamma(\pi)$.

Let $(U, u)$ be an adapted coordinate system on $E$, where $u=\left(x_{i}, u_{\alpha}\right)$. The induced coordinate system $\left(U^{1}, u^{1}\right)$ on $J^{1} E$ is defined by

$$
\begin{gathered}
U^{1}=\left\{J_{p}^{1} \phi: \phi(p) \in U\right\} \\
u^{1}=\left(x_{i}, u_{\alpha}, u_{\alpha}^{i}\right)
\end{gathered}
$$

where $x_{i}\left(J_{p}^{1} \phi\right)=x_{i}(p), u_{\alpha}\left(J_{p}^{1} \phi\right)=u_{\alpha}(\phi(p))$ and new functions

$$
u_{\alpha}^{i}: U^{1} \rightarrow R
$$

are specified by $u_{\alpha}^{i}\left(J_{p}^{1} \phi\right)=\frac{\partial \phi_{\alpha}}{\partial x_{i}}(p)$ and are known as derivative coordinates. Using those coordinate system, the following coordinate system are proposed to Minkowski 4_space. Let the bundle structure $\left(E_{2}^{4}, \pi, R\right)$ and the coordinates 
of the manifold $E_{2}^{4}$ are $\left(x_{1}, x_{2}, x_{3}, x_{4}\right)$, the coordinate of manifold $R$ is $(t)$. In addition the coordinates of the manifold $J^{1} E_{2}^{4}$ are $\left(t, x_{1}, x_{2}, x_{3}, x_{4}, \dot{x}_{1}, \dot{x}_{2}, \dot{x}_{3}, \dot{x}_{4}\right)$.

Then derivative coordinates are written as

$$
\dot{x}_{i}=\frac{\partial x_{i}}{\partial t}
$$

Let, we generate this total space and base space coordinates with split quaternions. Then coordinates of this space should be split quaternionic form to as following;

$$
\begin{gathered}
t=t_{0}+i t_{1}+j t_{2}+k t_{3}=t_{S}+t_{V} \\
q=q_{0}+q_{1} i+q_{2} j+q_{3} k=q_{i_{S}}+q_{i_{V}}, \quad 0 \leq i \leq 3 \\
\dot{q}=\dot{q}_{0}+\dot{q}_{1} i+\dot{q}_{2} j+\dot{q}_{3} k=\dot{q}_{i_{S}}+\dot{q}_{i_{V}}, \quad 0 \leq i \leq 3
\end{gathered}
$$

where $\dot{q}$ is the derivation of split quaternion parameter according to t time parameter. But, here time parameter is separated in two form; scalar part and vectoral part.

Also, derivation to time must be separated in to two part; derivation to scalar part and derivation to vectoral part. So, we can calculate following derivative coordinate;

$$
\begin{gathered}
\dot{q}=\left(\frac{d q_{0}}{d t_{S}}+\frac{d q_{1}}{d t_{S}} i+\frac{d q_{2}}{d t_{S}} j+\frac{d q_{3}}{d t_{S}} k\right)+\left(\frac{d q_{0}}{d t_{V}}+\frac{d q_{1}}{d t_{V}} i+\frac{d q_{2}}{d t_{V}} j+\frac{d q_{3}}{d t_{V}} k\right) \\
=\frac{d q_{0}}{d t_{S}}+\frac{d q_{0}}{d t_{V}}+\left(\frac{d q_{1}}{d t_{S}}+\frac{d q_{1}}{d t_{V}}\right) i+\left(\frac{d q_{2}}{d t_{S}}+\frac{d q_{2}}{d t_{V}}\right) j+\left(\frac{d q_{3}}{d t_{S}}+\frac{d q_{3}}{d t_{V}}\right) k \\
\dot{q}=\dot{q}_{0}+\dot{q}_{1} i+\dot{q}_{2} j+\dot{q}_{3} k \\
=\dot{q}_{i_{S}}+\dot{q}_{i_{V}}, \quad 0 \leq i \leq 3
\end{gathered}
$$

Then we can express the new split quaternionic form for shortness as follows;

$$
\left(t_{S}, t_{V}, q_{S}, q_{V}, \dot{q}_{S}, \dot{q}_{V}\right)
$$

\section{SPLIT QUATERNIONIC HAMILTONIAN SYSTEMS}

Minkowski 4_space is equivalent to 4_dimensional Euclid space, so it has a manifold structure can be built over the Minkowski 4_space too. With this cotangent manifold all geometrical structure for Hamiltonian systems can be defined for Minkowski space. Quaternions are generalization of complex numbers. Also, the quaternion algebra is the even subalgebra of the Clifford algebra of the 3_dimensional Euclidean space. The Clifford algebra $C l\left(E_{p}^{n}\right)=C l_{n-p, p}$ for the n- dimensional non degenerate vector space $E_{p}^{n}$.

The Minkowski 3_space $E_{1}^{3}$ is the Euclidean space $E_{1}^{3}$ provided with the inner product

$$
\langle u, v\rangle_{L}=-u_{1} v_{1}+u_{2} v_{2}+u_{3} v_{3}
$$

Also, the semi-Euclidean 4-space with 2-index represented with $E_{2}^{4}$. The inner product of this space is,

$$
\langle u, v\rangle_{E_{2}^{4}}=-u_{1} v_{1}-u_{2} v_{2}+u_{3} v_{3}+u_{4} v_{4}
$$

we can give the following definition using the inner product of;

1) If $\langle u, u\rangle_{E_{2}^{4}}<0$ then timelike vector

2) If $\langle u, u\rangle_{E_{2}^{4}}>0$ then spacelike vector

3) If $\langle u, u\rangle_{E_{2}^{4}}=0$ then lightlike (null) vector.

Similarly, an arbitrary curve $\alpha=\alpha(s)$ in $E_{2}^{4}$ can locally be spacelike curve, timelike curve or lightlike curve, if all of its velocity vectors $\alpha^{\prime}$ are respectively spacelike, timelike or lightlike, for every $s$.

$\tau$ is the set of all timelike vectors in $E_{2}^{4}$. For $\forall u \in \tau$; the set

$$
C(\vec{u})=\{\vec{x} \in \tau:\langle\vec{u}, \vec{x}\rangle<0\}=\left\{\vec{x} \in E_{2}^{4}: g(x-u, x-u)<0\right\}
$$

defined as timecone. [8] 
Theorem 2. The timelike vectors $\vec{x}$ and $\vec{y}$ in Minkowski 4 - space $E_{2}^{4}$ are in the same timecone,

$$
\langle\vec{x}, \vec{y}\rangle=-\|\vec{x}\|\|\vec{y}\| \cosh \theta
$$

and here $\theta$ is the Lorentz timelike angle between $\vec{x}$ and $\vec{y}$ vectors. [8]

The split quaternions are also identified with the $E_{2}^{4}$ space, and the pure split quaternions, which are the subspaces of the split quaternions, are identified by the Mikowski 3-space. Thus, it is possible to perform most of the operations in vector analysis using Lorentz inner and vector(exterior) products with split quaternions. [7]

In chapter 2, we defined the split quaternionic jet bundle coordinates. The following Liouville form can be defined accordingly.

Then, we can give following geometrical equations.

Definition 4. The Liouville form on split quaternionic structure or $E_{2}^{4}$-space,

$$
\begin{aligned}
\lambda= & -\dot{q}_{0} d q_{0}-\dot{q}_{1} d q_{1}+\dot{q}_{2} d q_{2}+\dot{q}_{3} d q_{3} \\
= & \sum_{i=0}^{3} \delta_{i}\left(\dot{q}_{i_{S}} d q_{i_{S}}+\dot{q}_{i_{V}} d q_{i_{V}}\right)
\end{aligned}
$$

here the notation $\delta_{i}$ is given by

$$
\delta_{i}=\left\{\begin{array}{cc}
-1, & i=0,1 \\
1, & i=2,3
\end{array}\right.
$$

with differentiating the Liouville form, with split quaternions we can obtain the canonical simplectic form as,

$$
\begin{gathered}
\phi=-d q_{0} \wedge d \dot{q}_{0}-d q_{1} \wedge d \dot{q}_{1}+d q_{2} \wedge d \dot{q}_{2}+d q_{3} \wedge d \dot{q}_{3} \\
=\sum_{i=0}^{3} \delta_{i}\left(d q_{i_{S}} \wedge d \dot{q}_{i_{S}}+d q_{i_{V}} \wedge d \dot{q}_{i_{V}}\right)
\end{gathered}
$$

We know that, the vector field $X_{H}$ is known as Hamiltonian vector field provided the symplectic form $i_{X_{H}} \phi=d H$.

Theorem 3. Split quaternionic Hamiltonian vector field on $R_{2}^{4}$ with bundle structure is expressed as

$$
X_{H}=\frac{\partial}{\partial t_{S}}+\frac{\partial}{\partial t_{V}}+\sum_{i=0}^{3}\left(\frac{\partial H}{\partial \dot{q}_{i}} \frac{\partial}{\partial q_{i}}-\frac{\partial H}{\partial q_{i}} \frac{\partial}{\partial \dot{q}_{i}}\right)
$$

Proof. Let,

$$
\begin{gathered}
X_{H}=\frac{\partial}{\partial t_{S}}+\frac{\partial}{\partial t_{V}}+\varepsilon_{0} \frac{\partial}{\partial q_{0}}+\varepsilon_{1} \frac{\partial}{\partial q_{1}}+\varepsilon_{2} \frac{\partial}{\partial q_{2}}+\varepsilon_{3} \frac{\partial}{\partial q_{3}} \\
+\mu_{0} \frac{\partial}{\partial \dot{q}_{0}}+\mu_{1} \frac{\partial}{\partial \dot{q}_{1}}+\mu_{2} \frac{\partial}{\partial \dot{q}_{2}}+\mu_{3} \frac{\partial}{\partial \dot{q}_{3}}
\end{gathered}
$$

and with calculating the symplectic form $i_{X_{H}} \phi=d H$, we can obtain following equalities,

$$
\begin{gathered}
i_{X_{H}} \phi=-\varepsilon_{0} d \dot{q}_{0}-\varepsilon_{1} d \dot{q}_{1}+\varepsilon_{2} d \dot{q}_{2}+\varepsilon_{3} d \dot{q}_{3} \\
+\mu_{0} d q_{0}+\mu_{1} d q_{1}-\mu_{2} d q_{2}-\mu_{3} d q_{3}
\end{gathered}
$$

and differential form of Hamiltonian energy function is defined as

$$
\begin{gathered}
d H=\frac{\partial H_{S}}{\partial t_{S}} d t_{S}+\frac{\partial H_{V}}{\partial t_{V}} d t_{V}-\frac{\partial H}{\partial q_{0}} d q_{0}-\frac{\partial H}{\partial q_{1}} d q_{1}+\frac{\partial H}{\partial q_{2}} d q_{2}+\frac{\partial H}{\partial q_{3}} d q_{3} \\
-\frac{\partial H}{\partial \dot{q}_{0}} d \dot{q}_{0}-\frac{\partial H}{\partial \dot{q}_{1}} d \dot{q}_{1}+\frac{\partial H}{\partial \dot{q}_{2}} d \dot{q}_{2}+\frac{\partial H}{\partial \dot{q}_{3}} d \dot{q}_{3}
\end{gathered}
$$

If this forms equalized each other the following solutions can be obtained,

$$
\frac{\partial H_{S}}{\partial t_{S}}=0, \frac{\partial H_{V}}{\partial t_{V}}=0, \mu_{0}=-\frac{\partial H}{\partial q_{0}}, \mu_{1}=-\frac{\partial H}{\partial q_{1}} \mu_{2}=-\frac{\partial H}{\partial q_{2}}, \mu_{3}=-\frac{\partial H}{\partial q_{3}}, \varepsilon_{0}=\frac{\partial H}{\partial \dot{q}_{0}}
$$




$$
\varepsilon_{1}=\frac{\partial H}{\partial \dot{q}_{1}}, \quad \varepsilon_{2}=\frac{\partial H}{\partial \dot{q}_{2}}, \quad \varepsilon_{3}=\frac{\partial H}{\partial \dot{q}_{3}}
$$

If we write this equalities in 3.4 then we can obtain Hamiltonian vector field as,

$$
X_{H}=\frac{\partial}{\partial t_{S}}+\frac{\partial}{\partial t_{V}}+\sum_{i=0}^{3}\left(\frac{\partial H}{\partial \dot{q}_{i}} \frac{\partial}{\partial q_{i}}-\frac{\partial H}{\partial q_{i}} \frac{\partial}{\partial \dot{q}_{i}}\right)
$$

Theorem 4. Hamiltonian energy equations with split quaternionic coordinates are:

$$
\begin{aligned}
& \frac{\partial H}{\partial \dot{q}_{i}}=\frac{d q_{i}}{d t_{S}} \text { and }-\frac{\partial H}{\partial q_{i}}=\frac{d \dot{q}_{i}}{d t_{S}} \\
& \frac{\partial H}{\partial \dot{q}_{i}}=\frac{d q_{i}}{d t_{V}} \text { and }-\frac{\partial H}{\partial q_{i}}=\frac{d \dot{q}_{i}}{d t_{V}}, 0 \leq i \leq 3
\end{aligned}
$$

Proof. Let $X_{H}$ is a vector field

$$
X_{H}=\frac{\partial}{\partial t_{S}}+\frac{\partial}{\partial t_{V}}+\sum_{i=0}^{3}\left(\frac{\partial H}{\partial \dot{q}_{i}} \frac{\partial}{\partial q_{i}}-\frac{\partial H}{\partial q_{i}} \frac{\partial}{\partial \dot{q}_{i}}\right)
$$

Suppose that integral curve belonging on $E_{2}^{4}$ is defined with split quaternions,

$$
\begin{aligned}
\alpha & : \quad I \subset R \rightarrow E_{2}^{4} \\
t & \rightarrow \quad \alpha(t)=\left(t_{S}, t_{V}, q_{i}, \dot{q}_{i}\right) \quad, \quad 0 \leq i \leq 3
\end{aligned}
$$

and it's velocity vector,

$$
\alpha^{\prime}(t)=\frac{\partial t_{S}}{\partial t_{S}} \frac{\partial}{\partial t_{S}}+\frac{\partial t_{V}}{\partial t_{V}} \frac{\partial}{\partial t_{V}}+\sum_{i=0}^{3}\left(\frac{d q_{i}}{d t_{S}} \frac{\partial}{\partial q_{i}}-\frac{d \dot{q}_{i}}{d t_{V}} \frac{\partial}{\partial \dot{q}_{i}}\right)
$$

With calculating the equation $X_{H}(\alpha(t))=\alpha^{\prime}(t)$, we can obtain following equalities,

$$
\begin{gathered}
\frac{\partial H}{\partial \dot{q}_{i}}=\frac{d q_{i}}{d t_{S}} \quad \text { and }-\frac{\partial H}{\partial q_{i}}=\frac{d \dot{q}_{i}}{d t_{S}} \\
\frac{\partial H}{\partial \dot{q}_{i}}=\frac{d q_{i}}{d t_{V}} \text { and }-\frac{\partial H}{\partial q_{i}}=\frac{d \dot{q}_{i}}{d t_{V}}, 0 \leq i \leq 3
\end{gathered}
$$

The equalities 3.9 are defined as Hamiltonian energy equations for split quaternionic space form.

\section{Example 1. (circle)}

In this study, movement actualized on a timelike curve, but we study with quaternions.

Also, this timelike curve parametrize with quaternions; so it has a scalar part and vectoral part jet coordinates include a derivative coordinate. This coordinate is derivativation coordinates of total manifolds to time parameter.

Studying with split quaternions, this derivative coordinates divided as to derivation of scalar time part and derivation of vectoral time part. For this reason, we want to define jet bundle coordinates with split quaternionic form for circle. For the circle parametrization,

$$
\alpha(\theta)=\left(t_{q}, r_{q} \cos \theta_{q}, r_{q} \sin \theta_{q}\right)
$$

the jet bundle coordinate is

$$
\left(t_{q}, r_{q} \cos \theta_{q}, r_{q} \sin \theta_{q}, \dot{r}_{q} \cos \theta_{q}, \dot{r}_{q} \sin \theta_{q}\right)
$$

More clearly,

$$
\left(t_{q}, r_{q} \cos \theta_{q}, r_{q} \sin \theta_{q}, \dot{r}_{q_{S}} \cos \theta_{q}, \dot{r}_{q_{V}} \cos \theta_{q}, \dot{r}_{q_{S}} \sin \theta_{q}, \dot{r}_{q_{V}} \sin \theta_{q}\right)
$$


Using this quaternionic circle bundle coordinates and simplifying equation 3.9, we can obtain Hamiltonian energy equation for this circle as follows;

$$
\begin{aligned}
& \text { 1) } \frac{\partial H_{S}}{\partial \dot{r}_{q_{S}} \cos \theta_{q}}=\frac{d r_{q_{S}} \cos \theta_{q}}{d t_{q_{S}}} \\
& \text { 2) } \frac{\partial H_{V}}{\partial \dot{r}_{q_{V}} \cos \theta_{q}}=\frac{d r_{q_{V}} \cos \theta_{q}}{d t_{q_{V}}} \\
& \text { 3) }-\frac{\partial H_{S}}{\partial \dot{r}_{q_{S}} \sin \theta_{q}}=\frac{d r_{q_{S}} \sin \theta_{q}}{d t_{q_{S}}} \\
& \text { 4) }-\frac{\partial H_{V}}{\partial \dot{r}_{q_{V}} \sin \theta_{q}}=\frac{d r_{q_{V}} \sin \theta_{q}}{d t_{q_{V}}}
\end{aligned}
$$

Now we calculate the common solution of this Hamiltonian equations. Here, the Hamiltonian function

$$
H\left(r_{q}, \theta_{q}\right)=H_{S}\left(r_{q}, \theta_{q}\right)+H_{V}\left(r_{q}, \theta_{q}\right)
$$

and the radius function $r_{q}=\left(r_{q_{S}}, r_{q_{V}}\right)$, the time function $t_{q}=\left(t_{q_{S}}, t_{q_{V}}\right)$. when the calculations are completed, Hamiltonian energy equations for circle are obtained as follows;

$$
\begin{aligned}
& H_{S}=\frac{\left(\dot{r}_{S}\right)^{2}}{2} \quad \text { scalar part of Hamiltonian energy } \\
& H_{V}=\frac{\left(\dot{r}_{V}\right)^{2}}{2} \quad \text { vectoral part of Hamiltonian energy }
\end{aligned}
$$

Also, split quaternionic Hamiltonian energy function can be written as,

$$
\begin{aligned}
H= & H_{S}+H_{V} \\
& \frac{1}{2}\left(\left(\dot{r}_{S}\right)^{2}+\left(\dot{r}_{V}\right)^{2}\right)
\end{aligned}
$$

\section{Conclusion and Discussion}

In this study, the improvement Hamiltonian energy equations have been proposed on Minkowski space $E_{2}^{4}$ with quaternionic bundle structure. Furthermore, in a different space model, jet bundle structure adapted to quaternions has been constituted. The following results can be driven from this study.

1) It is showed that the derivative coordinates for bundle structure has not an important role on the Hamiltonian energy equations. But, This derivative coordinates can adapted to quaternions.

2) Hamiltonian energy function is related by the constant value at time parameters. If the rate is constant, then the Hamiltonian energy is stable. This status, does not change in adapting to the quaternionic structure. In every algebraic space model, Hamiltonian energy is constant.

3) One of the encouraging results of this study is in any time interval, Hamiltonian energy of a movement particle can be seen in a static case. The conclusion of this study is similar to the study on Minkowski 4- space. Here, we divide time parameter in two part for quaternionic form.

4) Example also showed that, Hamiltonian energy values increase in a certain role and approach a fixed value. Energy is always perceived as stability.

5) Hamiltonian energy systems can be set up with quaternions. In Euclidian, space Hamiltonian energy equations is

$$
\frac{\partial x}{\partial t}=\frac{\partial H}{\partial \dot{x}}, \quad \frac{\partial \dot{x}}{\partial t}=-\frac{\partial H}{\partial x}
$$

Similarly in the studies on Minkowski 4-space

$$
\frac{\partial x_{i}}{\partial t}=\frac{\partial H}{\partial \dot{x}_{i}}, \quad \frac{\partial \dot{x}_{i}}{\partial t}=-\frac{\partial H}{\partial x_{i}}, \quad 1 \leq i \leq 4
$$

In this study, we found in the following way Hamiltonian energy equations

$$
\begin{aligned}
& \frac{\partial H}{\partial \dot{q}_{i}}=\frac{d q_{i}}{d t_{S}} \text { and }-\frac{\partial H}{\partial q_{i}}=\frac{d \dot{q}_{i}}{d t_{S}} \\
& \frac{\partial H}{\partial \dot{q}_{i}}=\frac{d q_{i}}{d t_{V}} \text { and }-\frac{\partial H}{\partial q_{i}}=\frac{d \dot{q}_{i}}{d t_{V}}, 0 \leq i \leq 3
\end{aligned}
$$


If we combine the vectorial part and scalar part, Hamiltonian energy equations 3.9 are similar with 4.1 and 4.2

As a result, Hamiltonian equations does not change in all spaces. Hamiltonian energy must be always in constant value. Results showed that Hamiltonian energy is stable, the movement is stable too. Time parameter is a reality for a space model. One of the encouraging results of this study is the scalar part of quaternion correspond the time parameter and naturally the result depends on this part.

\section{REFERENCES}

[1] C.Aycan, The Lifts of Euler-Lagrange and Hamiltonian Equations On The Extended Jet Bundles, D. Sc. Thesis, Osmangazi Univ. , Eskisehir, 2003.

[2] C. Aycan, S. Dagli, Improving Hamiltonian Energy Equation On The Kahler Jet Bundles, IJGMMP, V10 N3, March, 2013 .

[3] D. Brody, E. Graefe, On Complexified Mechanics and Coquaternions, Journal of Phys.A, V44, N7, 2011.

[4] M. Gogberashvily, O. Sakhelashvily; Geometrical Applications of Split Quaternions, Arxiv:1506.01012v2, 2015.

[5] M. De Leon and P.R. Rodrigues, Generalized Classical Mechanics and Field Theory, North-Holland Mathematics Studies, Vol. 112, (Elsevier Science Publishing, Amsterdam, 1991.

[6] M. Özdemir, A.A Ergin, Some Geometric Applications of Timelike Quaternions, Int. Conf. Jonhjean Math. Soc., V. 16, pp 108-115, 2005.

[7] M. Özdemir, A.A Ergin, Rotations with unit timelike quaternions in Minkowski 3-space, Journal of Geometry and Physics, V. 56, pp 322-336, 2006.

[8] S. Dagli, The Jet Structure and Mechanical Systems On Minkowski 4-Space, PhD Thesis,Pamukkale Univ., Denizli, 2012.

[9] G. Sardanashvily, "Hamiltonian time-dependent mechanics”, Jour. Of Math. Phys., 39-(5), (1998).

[10] G.Sardanashvily, and O. Zakharov, "On Application Of The Hamilton Formalism in Fibred Manifolds To Field Theory”, Dif. Geo. And İts App., 3, 245-263, (1993). 